\title{
Structural characterization of complexes prepared with glycerol monoestearate and maize starches with different amylose contents
}

\author{
M.C. Garcia ${ }^{a}$, M.A. Pereira-da-Silva ${ }^{\text {b,c }}$, S. Taboga ${ }^{a}$, C.M.L. Franco ${ }^{a, *, 1}$ \\ a São Paulo State University, UNESP, São José do Rio Preto, SP, Brazil \\ b São Paulo University, USP, São Carlos, SP, Brazil \\ ${ }^{\mathrm{c}}$ Central Paulista University Center, UNICEP, São Carlos, SP, Brazil
}

\section{A R T I C L E I N F O}

\section{Article history:}

Received 9 November 2015

Received in revised form 13 April 2016

Accepted 18 April 2016

Available online 19 April 2016

\section{Keywords:}

Inclusion complex

Emulsifier

V-amylose

Amylopectin

Microscopy

\begin{abstract}
A B S T R A C T
Morphology and different structural features of $\mathrm{V}$-amylose complexes prepared with different concentrations ( $1 \%, 2 \%, 3 \%$ ) of glycerol monostearate (GMS) and normal maize (NMS), waxy maize (WMS), and high amylose maize (HAMS) starches were evaluated using X-ray diffraction, differential scanning calorimetry, scanning electronic microscopy (SEM), atomic force microscopy (AFM), and transmission electronic microscopy (TEM). There was inclusion complex formation between all starches and GMS regardless of emulsifier concentration, with exception of WMS-2\%GMS and WMS-3\%GMS samples. All of the inclusion complexes displayed a V-type crystalline pattern and endothermic dissociation peaks between 115 and $120^{\circ} \mathrm{C}$. They also displayed faceted crystalline structures with a tendency of the crystals to aggregate and form agglomerates of various sizes. TEM images of the complexes showed an aggregated strand structure interwoven with the GMS. Emulsifier and amylose quantities directly influenced complex formation. At high GMS concentrations, there was higher tendency of emulsifier to self-associate rather than form complexes with amylose.
\end{abstract}

() 2016 Elsevier Ltd. All rights reserved.

\section{Introduction}

Amylose-lipid complexes, which are responsible for important functional interactions in food systems, can be found naturally in starch or formed during its gelatinization in the presence of lipids and/or emulsifiers (Putseys, Lambert, \& Delcour, 2010). In native starch, the amylose-lipid complex is in an amorphous state, but its crystallization becomes possible when the starch is heated in water (Morrison, Law, \& Snape, 1993). Meanwhile, complexing with added lipids requires amylose to be accessible and to have enough mobility to undergo conformational changes (Conde-Petit, Escher, \& Nuessli, 2006), which result in a single, left-handed helix with a V-amylose crystalline pattern (Putseys et al., 2010).

The amylose-lipid complex can exist in two forms according to its complexing temperature. Type I is formed at a low complexing temperature $\left(<60^{\circ} \mathrm{C}\right)$ and presents a low dissociation temperature

\footnotetext{
* Corresponding author at: UNESP, São Paulo State University, Department of Food Engineering and Technology, São José do Rio Preto, SP, Brazil.

E-mail addresses: marinacosta16@gmail.com (M.C. Garcia), maps@ifsc.usp.br (M.A. Pereira-da-Silva), taboga@ibilce.unesp.br (S. Taboga), celia@ibilce.unesp.br (C.M.L. Franco).

1 Rua Cristovão Colombo, 2265, CEP: 15054-000 São José do Rio Preto, SP, Brazil.
}

$\left(<100^{\circ} \mathrm{C}\right)$, while type II or semicrystalline is formed at high temperature $\left(>90^{\circ} \mathrm{C}\right)$ and has a high dissociation temperature $\left(>100^{\circ} \mathrm{C}\right)$ (Gelders, Vanderstukken, Goesaert, \& Delcour, 2004). The type II complex displays V- conformation, while the type I has been described as amorphous (Galloway, Biliaderis, \& Stanley, 1989). However, Gelders et al. (2004) showed a semicrystalline V-pattern for type I complexes analyzed with $10 \%$ moisture, while a very weak $\mathrm{V}$-pattern was obtained when the complexes were wet. Indeed, the $\mathrm{X}$-ray spectrum appears to be highly dependent upon the conditions in which it is obtained. Cheetam \& Tao (1998) reported that hydration induces an increase in the degree of crystallinity of starch but does not change the transition of crystal type.

A distinction is made between the two possible type II amyloselipid complexes based on their degrees of crystallinity. They are referred to as type IIa and IIb complexes. The type IIb has a melting temperature that is higher than that of the type Ila, but both melting temperatures are above $100^{\circ} \mathrm{C}$ (Karkalas, Morrison, \& Pethrick, 1995).

The structural and physicochemical characteristics of amyloselipid complexes vary according to the types of lipids and starches involved and conditions under which the complex is formed. The amylose content and the chain length of starch, as well as fatty acid chain length and the thermal treatment conditions used in the process are important factors that determine the inclusion complex 
formation (Chang, He, \& Huang, 2013a; Fanta, Felker, Shogren, \& Salch, 2006; Seo, Kim, \& Lim, 2015; Villwock, Eliasson, Silverio, \& Bemiller, 1999; Zhou, Ren, Zhang, Yoo, \& Lim, 2013). Starches with a high amount of amylose have more molecules available to interact with the lipid and allow more complexes to be formed while long amylose chains favor more crystalline and stable complex formation (Exarhopoulos \& Raphaelides, 2012; Gelders et al., 2004; Seo et al., 2015; Zhou et al., 2013). However, conformational disorders may occur if the amylose chains are too long, resulting in faults in the crystal structure (Gelders et al., 2004, Putseys et al., 2010). On the other hand, the lipids with long chain length are best for complex formation because they allow more hydrophobic interactions with the interior of the helix (Kawai, Takato, Sasaki, \& Kajiwara, 2012; Putseys et al., 2010; Tang \& Copeland, 2007).

Amylopectin is limited in its ability to form V-type complexes due to its highly branched structure. However, long branch chains of this molecule can also form helices that have been shown to complex with lipids (Chang et al., 2013a; Eliasson, 1994; Villwock et al., 1999). Inclusion complex formation between amylopectin and lipids has been shown by differential scanning calorimetry, Xray diffraction (Chang et al., 2013a; Garcia \& Franco, 2015; Villwock et al., 1999), and light microscopy (Chang et al., 2013a). However, complexing between waxy starch and lipids is less easily discernible than in maize starches with high amylose contents (Chang et al., 2013a; Exarhopoulos \& Raphaelides, 2012; Villwock et al., 1999).

Starch-lipid inclusion complexes have been characterized mainly by their thermal properties and crystallinity (Chang et al., 2013a; Chang, He, \& Huang, 2013b; Exarhopoulos \& Raphaelides, 2012; Vasiliadou, Raphaelides, \& Papastergiadis, 2015; Zhang, Huang, Luo, \& Fu, 2012). A few reports have also shown the structural characteristics of these complexes using microscopic techniques such as scanning electron microscopy (SEM) (Exarhopoulos and Raphaelides, 2012; Fanta, Felker, \& Shogren, 2002; Fanta et al., 2006; Zabar, Lesmes, Katz, Shimoni, \& BiancoPeled, 2009; Zabar, Lesmes, Katz, Shimoni, \& Bianco-Peled, 2010), atomic force microscopy (AFM) (Lalush, Bar, Zakaria, Eichler, \& Shimoni, 2005; Lesmes, Cohen, Shener, \& Shimoni, 2009; Zabar et al., 2010), and transmission electron microscopy (TEM) (Godet, Bouchet, Colonna, Gallant, \& Buléon, 1996; Richardson, Kidman, Langton, \& Hermansson, 2004). These techniques have been applied to help elucidate the attributes of V-amylose complexes on the micro and nano scales and may provide new information even to previously studied starch systems.

SEM has revealed V-amylose complexes as faceted crystalline structures or spherocrystalline particles (Fanta et al., 2002; Fanta et al., 2006; Zabar et al., 2009, 2010). Previous reports have shown amylose-fatty acid complexes that are organized in crystalline lamellae as spherulites, which tend to aggregate in a radial direction from a central nucleus (Fanta et al., 2002; Fanta et al., 2006; Zabar et al., 2010). The increase in the degree of unsaturation of the fatty acid may lead to the formation of poorly defined complexes and decrease their thermal stability (Zabar et al., 2009). On the other hand, Fanta et al. (2006) reported that besides the influence of the fatty acid structure on the amylose helix conformation, the experimental conditions are also important to control spherulite morphology. Lalush et al. (2005) have also reported that the experimental conditions for inclusion complex formation affect the shape, size, and structural organization of the complexes.

Lalush et al. (2005), Lesmes et al. (2009), and Zabar et al. (2010) analyzed V-amylose complexes in AFM and reported that they are submicron-sized spheroids organized into packed lamellae that form aggregates that are microscopic in size. These aggregates were understood to be comprised of small, $\sim 50-100-n m$ spherulites in
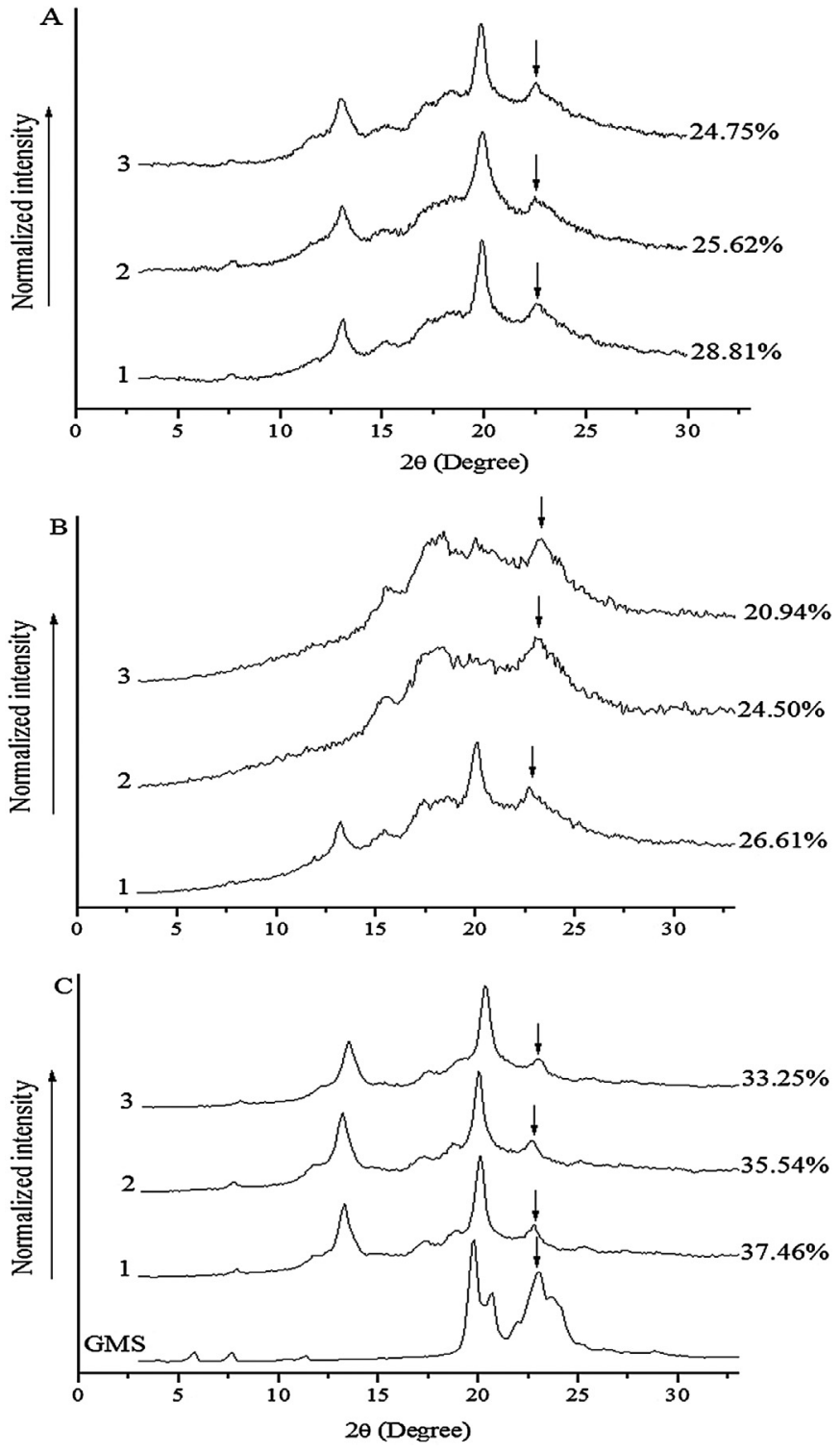

Fig. 1. X- ray pattern of the GMS, and X-ray pattern and relative crystallinity (\%) of the starch-lipid complexes prepared with maize starches with different amylose contents and GMS in different concentrations: A: NMS, B: WMS, C: HAMS. (1): 1\% GMS; (2): 2\% GMS; (3): 3\% GMS.

lamellae that are a few microns in length and $\sim 10 \mathrm{~nm}$ thick, and a few other poorly-defined structures.

Morphology and the crystal thickness of amylose-fatty acid complexes were studied using TEM (Godet et al., 1996). Lamellar arrangements formed by the alternation of crystalline and amorphous areas were observed; the dimensions were found to be dependent on amylose DP and fatty acid chain length. Crystal thickness increased as the amylose and fatty acid chain lengths increased, reaching $4.5 \mathrm{~nm}$. On the other hand, Richardson et al. (2004) investigated the effect of different kinds of emulsifiers on network formation and aggregation of amylose and starch gels using TEM. They observed that, at certain concentrations, the emulsifiers played an important role in amylose aggregation. The normal amylose network consists of thin strands. However, amylose was found to aggregate into thicker and more rigid strands at moderate emulsifier concentrations and to gather into unstructured, spherical aggregates at high emulsifier concentrations.

In a recent paper Garcia \& Franco (2015) reported the effect of different glycerol monostearate (GMS) concentrations on both 

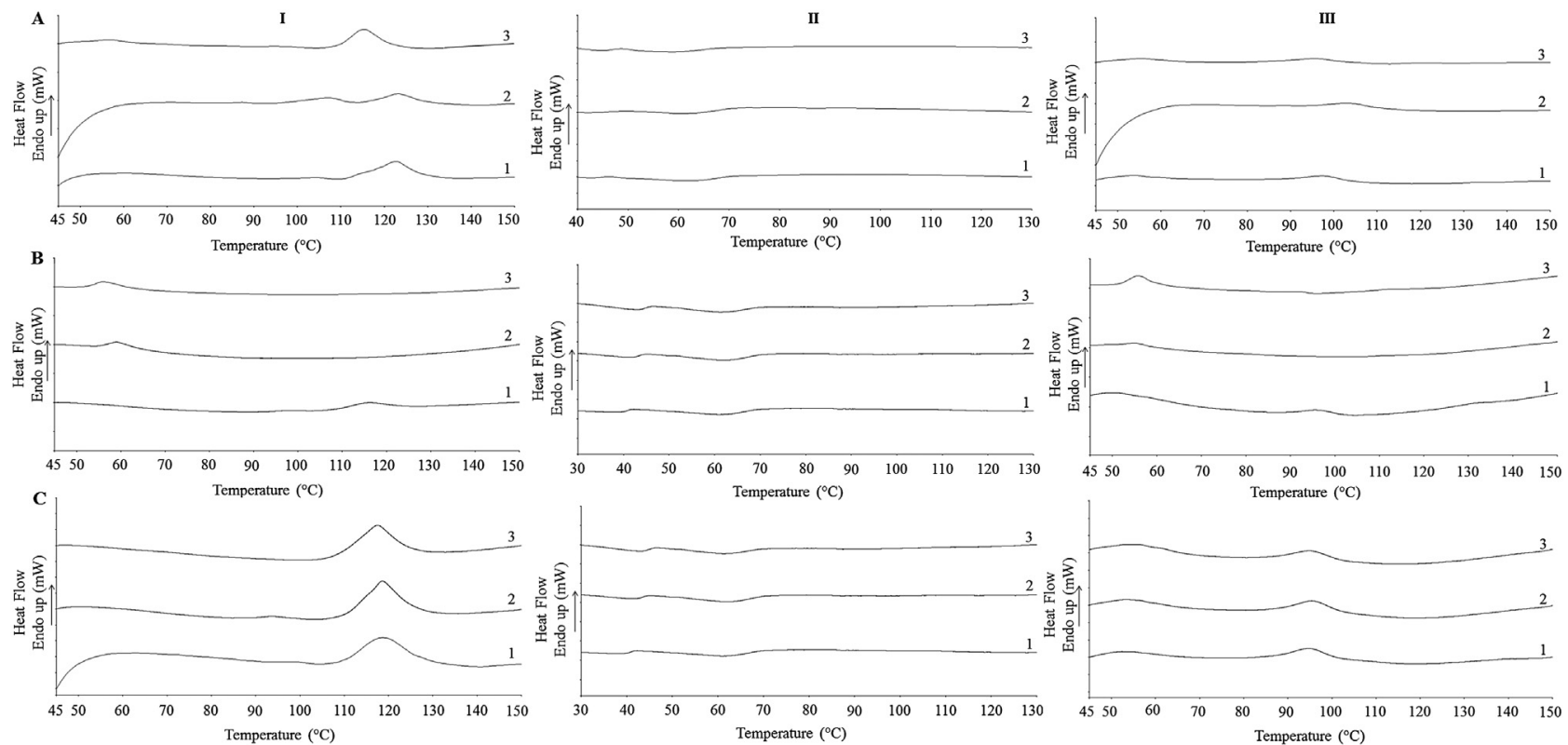

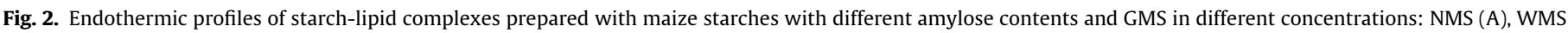
(B), HAMS (C). (1) 1\%, (2) 2\%, (3) 3\%; (I) 1st heating, (II) cooling, (III) 2nd heating.

complex formation and the gelatinization behavior of maize starches with different amylose contents. The quantities of the emulsifier and amylose had a direct influence on the complex formation. Concentration of the GMS lower than $2 \%$ was necessary to complex with available amylose in normal maize starch, while at least 3\% GMS was necessary in high amylose maize starch.

Systematic studies combining molecular level investigations with nanostructure and microscopic characterization of V-amylose have been reported. However, the data are not yet sufficient to allow a full understanding of the molecular and structural characteristics of the inclusion complexes because their formation depends on the starch and lipid types, amounts and chain lengths of amylose and lipids, degree of unsaturation of the lipids, and gelatinization conditions. The aim of this study was to systematically investigate the effect of the GMS and amylose amounts on the molecular organization and structure of V-amylose complexes. Inclusion complexes were prepared using different concentrations of a nonionic emulsifier with a long carbon chain (GMS) and normal maize, waxy maize and high amylose maize starches. X-ray diffraction and DSC were used to study the molecular organization of the complexes, while SEM, AFM, and TEM evaluated their microand nano-structures. The effect of the GMS concentration on the complex formation was also evaluated.

\section{Materials and methods}

\subsection{Materials}

Normal maize (Amilogill 2100), waxy maize (Waxy),and high amylose maize (AmyloGel 03003) commercial starches were provided by Cargill Agricola S/A (São Paulo, Brazil). In this study, these starches have been coded as NMS (normal maize starch), WMS (waxy maize starch), and HAMS (high amylose maize starch). The amylose content of the starches has been determined and reported in a previous study (Garcia and Franco, 2015): 25\%, 1\%, and 49.8\% amylose in NMS, WMS, and HAMS, respectively.

Glycerol monostearate (GMS $>99 \%$ pure) was acquired from Alfa Aesar Johnson Matthey Company (Ward Hill, USA). The pancreatin, amyloglucosidase, and invertase enzymes were obtained from Sigma Chemical Co. (St. Louis, USA).

\subsection{Preparation of starch-GMS complex}

The preparation of the starch-GMS complex was based on the procedure described by Galloway, Biliaderis, \& Stanley (1989). Samples of $4 \mathrm{~g}$ of starch were dispersed in $25 \mathrm{~mL}$ of hot dimethyl sulfoxide (DMSO). This solution was then diluted in $375 \mathrm{~mL}$ of boiling deionized water and stirred continuously for $30 \mathrm{~min}$ in a double boiler. Glycerol monostearate $(1-3 \% \mathrm{w} / \mathrm{v})$ was dissolved in $20 \mathrm{~mL}$ of preheated ethanol $\left(65^{\circ} \mathrm{C}\right)$, added dropwise to the starch solution, and stirred continuously. The mixture was incubated at $90^{\circ} \mathrm{C}$ for $4 \mathrm{~h}$ with stirring every $30 \mathrm{~min}$ and slowly cooled at room temperature for $20 \mathrm{~h}$ in a thermal bottle. The complexes were recovered by centrifuging at $15000 \mathrm{~g}$ for $20 \mathrm{~min}$ at $6^{\circ} \mathrm{C}$, washed three times with $200 \mathrm{~mL}$ distilled water, centrifuged at $15000 \mathrm{~g}$ for $15 \mathrm{~min}$ at $6^{\circ} \mathrm{C}$, and lyophilized.

\subsection{X-ray diffraction}

Starch-GMScomplex samples were stored for 10 days at $25^{\circ} \mathrm{C}$ and at $90 \%$ relative humidity in a desiccator containing a saturated solution of $\mathrm{BaCl}_{2}$ and $1 \%$ sodium azide. The X-ray diffraction patterns of the samples were determined using a bench top Xray diffractometer (MiniFlex 300, Rigaku, Tokyo, Japan) with $\mathrm{Cu}$ $K \alpha$ monochromatic radiation $(\lambda=0.1542 \mathrm{~nm})$. The scanning speed was $2 \circ \mathrm{min}$ at $30 \mathrm{kV}$ and $10 \mathrm{~mA}$. Relative crystallinity was quantitatively estimated based on the relationship between the peak and total areas according to the method described by Nara \& Komiaya (1983).

\subsection{Thermal properties}

The thermal properties of the complexes were analyzed using a differential scanning calorimeter (DSC-Pyris 1, Perkin Elmer, Waltham, USA). Complex samples ( $4 \mathrm{mg}$, dry basis) were weighed in stainless steel pans, mixed with deionized water $(12 \mu \mathrm{L})$, and sealed. The sealed pans were kept at room temperature for $12 \mathrm{~h}$ to equilibrate. The analyses were carried out in three steps: 1 . heating from 25 to $200^{\circ} \mathrm{C} ; 2$.cooling from 200 to $25^{\circ} \mathrm{C}$; 3. reheating from 25 to $200^{\circ} \mathrm{C}$. All steps were carried out at a rate of $20^{\circ} \mathrm{C} / \mathrm{min}$. An empty stainless steel pan was used as a reference. Transition tempera- 


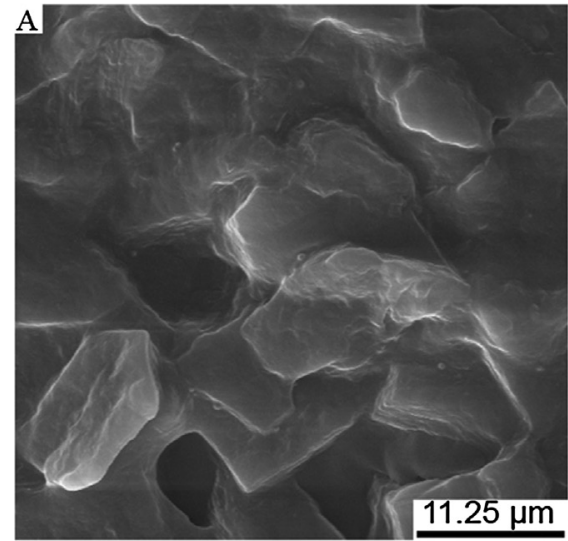

$\mathrm{D}$

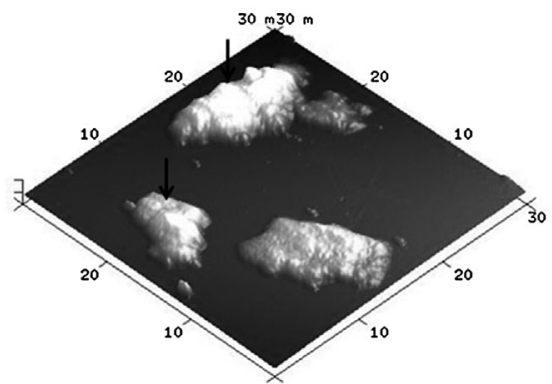

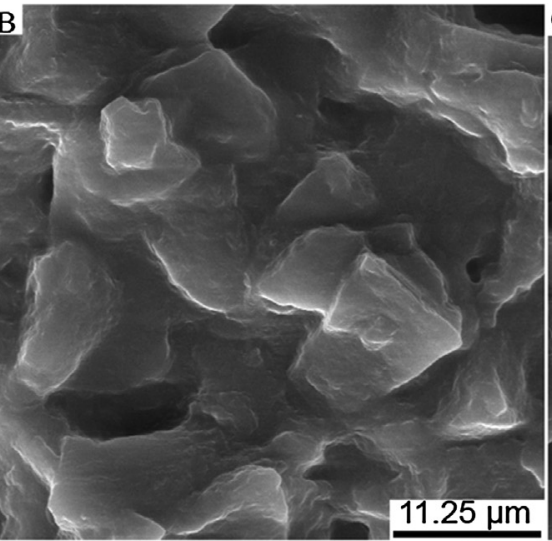

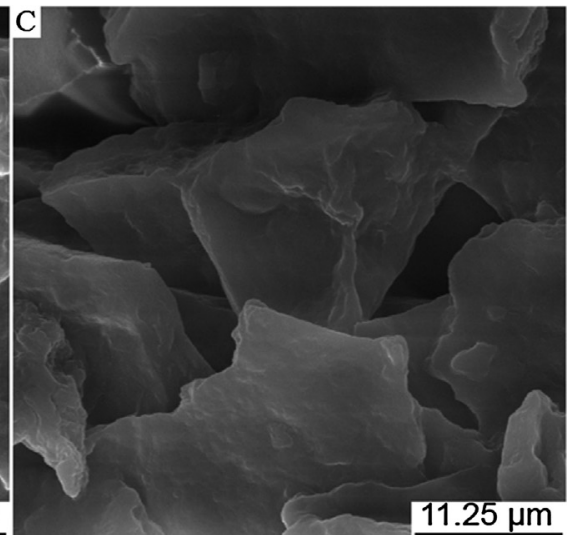

E
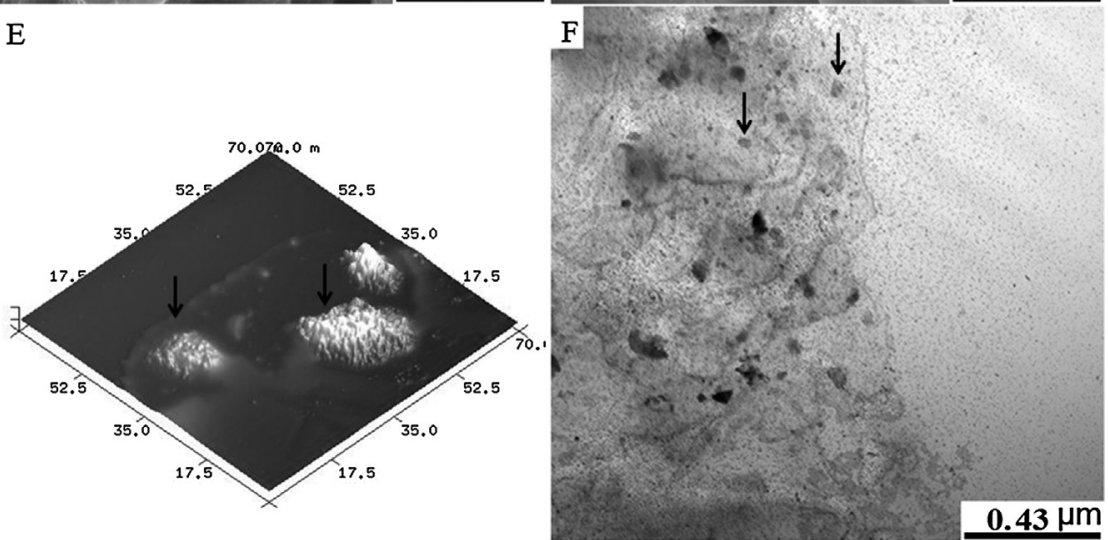

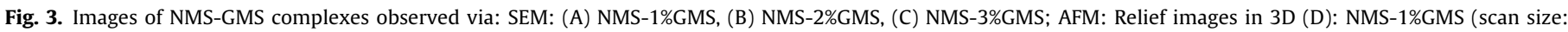
$30 \mu \mathrm{m} \times 30 \mu \mathrm{m}$ ); (E): NMS-3\%GMS (scan size: $70 \mu \mathrm{m} \times 70 \mu \mathrm{m}$ ); TEM: (F) NMS-1\%GMS.

tures (initial, peak, and final) and enthalpy variation $(\Delta H)$ of the starch-GMS complexes during the heating, cooling, and reheating cycles were determined using the Pyris 1software (Perkin Elmer, Waltham, USA).

\subsection{Scanning electron microscopy}

The starch-GMS complex was rinsed with absolute ethanol and mounted on a metal plate covered with carbon double-sided adhesive tape to which a 20 -nm gold layer was applied. The complex was seen under a scanning electron microscope (DSM 960, Zeiss, Oberkochen, Germany) operating at an accelerating voltage of $20 \mathrm{kV}$.

\subsection{Atomic force microscopy}

An atomic force microscope (Label Bruker, Icon model, Santa Barbara, USA) was used for analyzing starch-GMS complex. Aliquots of NMS-GMS, WMS-GMS and HAMS-GMS samples in aqueous suspension $(5 \mathrm{mg} / \mathrm{mL}$ ) were directly deposited onto a mica slide and dried at room temperature overnight. The mica slides were covered to prevent any particles from the air from coming in contact with the sediment on the mica. The mica slides containing the samples were placed on the AFM table, and the scanning was performed in intermittent contact with the silicon rectangular cantilever, with spring constants of $40 \mathrm{~N} / \mathrm{m}$ and an operating frequency of $330 \mathrm{kHz}$. Scan areas ranged from $2 \mu \mathrm{m}$ to $70 \mu \mathrm{m}$. AFM images (3D, relief, phase, and amplitude) were processed using the NanoScope Analysis software, which was also used to calculate the diameter, height, and roughness measures of the crystals.

\subsection{Transmission electron microscopy}

The complex samples were prepared based on the procedure described by Richardson et al. (2004), with modifications. The complex samples were fixed with $2.5 \%$ glutaraldehyde and $0.2 \%$ ruthenium red in $0.1 \mathrm{M}$ phosphate buffer with $\mathrm{pH} 7$ at $17^{\circ} \mathrm{C}$ for $24 \mathrm{~h}$. After being washed with the same buffer, the samples were dehydrated using various steps with increasing acetone concentrations (50\%, 75\%, 95\%, and 99.5\%) and embedded in Araldite 502 Resin (Electron Microscopy Sciences, Hatfield, USA). Ultrathin sections ( $50 \mathrm{~nm}$ ) were cut using diamond knives and stained with $2 \%$ uranyl acetate for $30 \mathrm{~min}$, followed by $2 \%$ lead citrate in sodium hydroxide for $10 \mathrm{~min}$. Images were taken with a LEO 906 transmission electron microscope (Zeiss, Cambridge, UK) at an accelerating voltage of $80 \mathrm{kV}$.

\subsection{Statistical analysis}

The DSC and XRD experiments were performed in triplicate and the data were compared through the analysis of variance (ANOVA) followed by the Tukey test $(p \leq 0.05)$. The measures from microscopic images were performed in duplicate and the data were compared through Student $t$ test $(\mathrm{p} \leq 0.05)$. The software Statistica for Windows (version 7.0, Statsoft, Tulsa, USA) was used for all analyses.

\section{Results and discussion}

\subsection{X-Ray diffraction}

X-ray diffraction was used in order to determine whether starch-GMS complex formation has occurred. Besides the starch- 
GMS samples, a spectrum of pure GMS was also obtained (Fig. 1), which displayed two prominent intensity peaks at 19.7 and 23.0 diffraction degrees. Carrillo-Navas, Pérez-Alonso, Fouconnier, Vernon-Carter, \& Alvarez-Ramírez (2014) also reported the peaks at $19.97^{\circ}$ and $23.38^{\circ}$ at $2 \theta$ corresponding to pure GMS.

All of the starches formed inclusion complexes with GMS, as confirmed by V-type patterns displayed in difractograms of the samples (Fig. 1). The V-pattern was inferred from three main peaks corresponding to the Bragg angles $2 \theta$ at $\sim 7^{\circ}, \sim 13^{\circ}$, and $\sim 20^{\circ}$ (Chang et al., 2013a; Galloway et al., 1989; Lesmes et al., 2009; Vasiliadou et al., 2015; Zabar et al., 2010).

Diffraction peaks at $\sim 22.8^{\circ}$ at $2 \theta$, which corresponded to GMS aggregates, were observed at higher or lower resolution for all the samples. The diffractograms of the WMS-GMS samples, especially at 2 and 3\% GMS, displayed this peak at higher resolution. Lesmes et al. (2009), Tang \& Copeland (2007), Zabar et al. (2009) and Zabar et al. (2010) also reported peaks at $\sim 21^{\circ}$ and $\sim 24^{\circ}$ at $2 \theta$ and attributed them to an excess of stearic acid molecules that could be physically trapped between amylose double helices within the matrix and could have therefore self-associated. Above a certain concentration, lipids with long carbon chains have low solubility and a tendency to self-associate in micellar structures rather than forming complexes with amylose helices (Tang and Copeland, 2007; Zhang et al., 2012). The critical micelle concentration of GMS is $4.0 \mathrm{wt} \%$ in oils (Ghosh and Rousseau, 2009).

Both the amylose content and GMS concentration affected complex formation. Regardless of GMS concentration, the X-ray diffractograms of the NMS-GMS and HAMS-GMS complexes clearly displayed the peaks at $\sim 7^{\circ}, \sim 13^{\circ}$, and $\sim 20^{\circ}$ at $2 \theta$. These peaks were taller and clearer in HAMS-GMS complexes than in NMS-GMS complexes (Fig. 1A and C). In the case of WMS-GMS samples, the diffractograms did not display a good resolution (Fig. 1B). However, the V-type pattern could be observed in the diffractogram of the WMS-1\%GMS complex (Fig. 1B1). These results are correlated with the different amylose contents of the starches. On the other hand, the increase in GMS concentration slightly decreased the resolution of the peaks at $7^{\circ}, 13^{\circ}$, and $20^{\circ}$ and slightly increased the resolution of the peak at $\sim 22.8^{\circ}$ at $2 \theta$ in diffractograms of the NMS-GMS complexes (Fig. 1A). There was a decrease of $\sim 14 \%$ in the relative crystallinity of the NMS-GMS complexes when the GMS concentration increased from $1 \%$ to $3 \%$. These results suggest that, at higher concentrations there was a higher tendency for the emulsifier to self-associate, which diminished its capacity to complex with amylose. It is possible that the critical micellar GMS concentration for the formation of the NMS-GMS complex is close to $1 \%$.

Only the diffractogram of WMS-1\%GMS displayed the peaks of the V-pattern. This result suggests that inclusion complex formation occurred between few amylose molecules and/or between the amylopectin long branch chains of the WMS and 1\% GMS (Fig. 1B1). Higher GMS concentrations drastically reduced these peaks and increased the resolution of the peak at $\sim 22.8^{\circ}$ (Fig. 1B2 and B3). Because of the few amylose molecules and long branch chains of amylopectin there was a high tendency to self-association of GMS molecules.

The diffractograms of the HAMS-GMS complexes exhibited peaks with excellent resolutions at all GMS concentrations due to the high amylose content (49.8\%) of the HAMS (Fig. 1C1-C3). This result indicated that, even with $3 \%$ GMS amylose was still available to complex with HAMS. However, the relative crystallinity also decreased as the GMS concentration increased, a result that confirms the emulsifier's tendency toward self-association. Chang et al. (2013a) and Exarhopoulos \& Raphaelides (2012) reported that the relative crystallinity and resolution of the peaks in difractograms of the starch-lipid complexes were mainly influenced by amylose con- tent, fatty acid chain length, and the type of the thermal treatment used.

\subsection{Differential scanning calorimetry}

All of the starch-lipid complex thermograms revealed endothermic peaks between 115 and $120^{\circ} \mathrm{C}$ in the heating cycle (Fig. 2-I). These values corresponded to amylose-GMS complex dissociation.The exception was the WMS-2\%GMS and WMS-3\%GMS samples, which did not display complex dissociation peaks (Fig. 2-I, $\mathrm{B} 2$ and B3). These results agree with those obtained from the X-ray diffraction (Fig. 1B).

The high dissociation temperatures of the complexes $\left(t_{c x} \sim 118^{\circ} \mathrm{C}\right)$ allowed for their classification as V-patterns with an IIb polymorphism, which consist of crystals with well-defined structures (Karkalas et al., 1995). These results may be related to the chain lengths of amylose and GMS. According to Kawai et al. (2012), the higher the peak temperature, the higher the complex helical length is and, consequently, the greater its physical stability. The emulsifier chain length is also an important factor that affects both the characteristics and the extent of complex formation. Emulsifiers with long carbon chains, as is the case of GMS, provide greater thermostability to V-type complexes (Godet et al., 1995; Mira, Persson, \& Villwock, 2007; Putseys, Liesbeth, Lamberts, Goesaert, \& Delcour, 2009).

The increase in GMS concentration reduced the peak temperature $\left(t_{c x}\right)$ in NMS-GMS complexes from $120.65^{\circ} \mathrm{C}$ in the NMS-1\%GMS complex to $115.84^{\circ} \mathrm{C}$ in the NMS-3\%GMS complex (Fig. 2-I, A1 and A3), indicating the lower stability of the complexes when the GMS concentration increased. In addition, two endothermic peaks above $100^{\circ} \mathrm{C}$ were observed in the NMS-2\%GMS complex (Fig. 2-I, A2). This result suggests that, at this concentration, both type-Ila and type-IIb complexes were formed. The enthalpy $\left(\Delta \mathrm{H}_{\mathrm{Cx}}\right)$ of the NMS-GMS complexes decreased as the GMS concentration increased; enthalpy values decreased from $7.66 \mathrm{~J} / \mathrm{g}$ in the NMS$1 \%$ GMS to $5.58 \mathrm{~J} / \mathrm{g}$ in the NMS-3\%GMS. These results indicate that $1 \%$ GMS was sufficient to complex with available amylose in NMS, and that, above this concentration, GMS was more likely to selfassociate, as revealed by the X-ray diffractograms (Fig. 1A).

A small peak between 50 and $60^{\circ} \mathrm{C}$ was observed in the NMS3\%GMS, WMS-2\%GMS and WMS-3\%GMS complexes (Figs. 2-I, A3, B2 and B3). This peak probably corresponded with self-associated GMS dissociation (Chang et al., 2013a; Mira et al., 2007).

The peak temperatures and enthalpy $\left(118^{\circ} \mathrm{C}\right.$ and $\left.14.9 \mathrm{~J} / \mathrm{g}\right)$ of the HAMS-GMS complexes did not change, regardless of GMS concentration. This result was likely due to the sufficient amylose amount for complexing with all GMS (Figs. 2-I, C1-C3). In a previous study (Garcia \& Franco, 2015), we reported that the quantities of the emulsifier and amylose directly influence in vitro complex formation. GMS concentrations lower than $2 \%$ were found to be necessary for the emulsifier to complex with available amylose in NMS, while at least 3\% GMS was necessary in the case of HAMS.

The $\mathrm{t}_{\mathrm{cx}}$ and $\Delta \mathrm{H}_{\mathrm{cx}}$ of the NMS-GMS and WMS-GMS complexes were lower than those of the HAMS-GMS complexes. In addition, with the increase in GMS concentrations (to 2\% and 3\% GMS), there were decreases in temperatures and enthalpies of the NMS-GMS complexes. However, the HAMS-GMS complexes did not display changes in these parameters as GMS concentration increased. These results indicate that more HAMS-GMS complexes are formed and are more thermostable than NMS-GMS and WMS-GMS complexes due to the greater amount of amylose available. Kawai et al. (2012) reported that enthalpy is a measure of both the amount and the crystalline order of the complex.

The exothermic peaks relative to recrystallization of the complexes, observed in DSC cooling curves (Fig. 2-II), occurred at temperatures that were lower than those of the fusion peaks. 

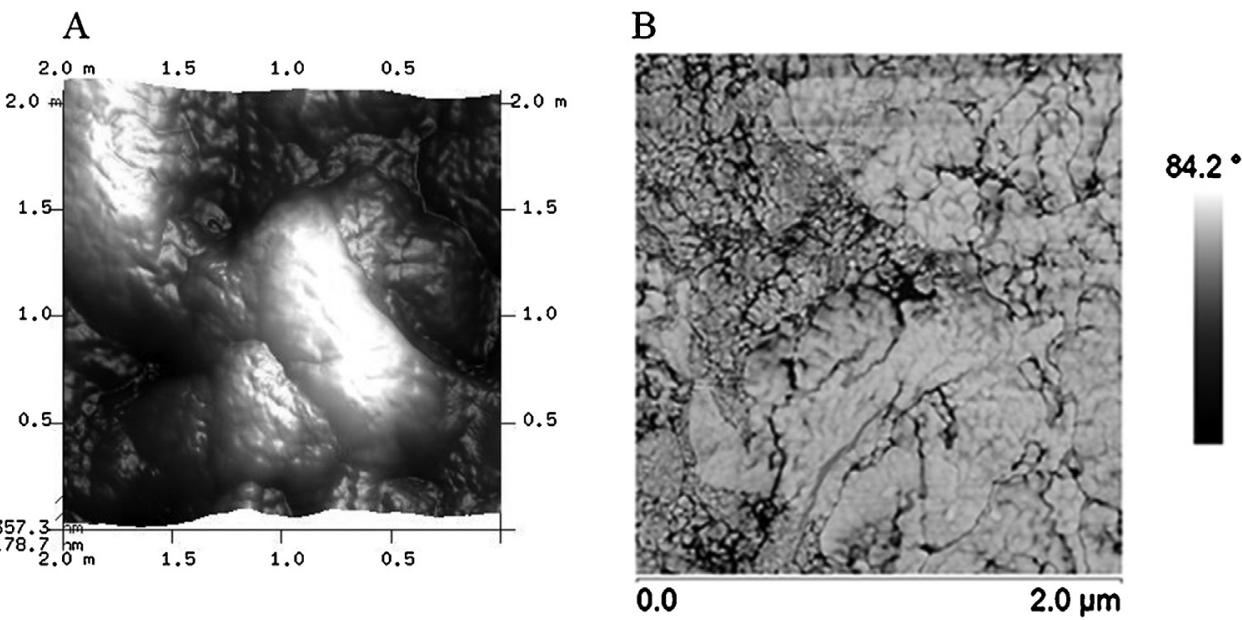

Fig. 4. AFM Images of NMS-3\%GMS (scan size $2.0 \mu \mathrm{m} \times 2.0 \mu \mathrm{m}$ ): (A) Relief images in 3D, (B) phase image.

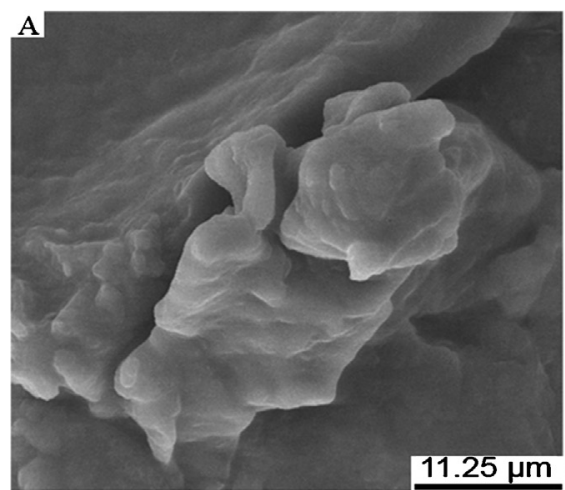

D

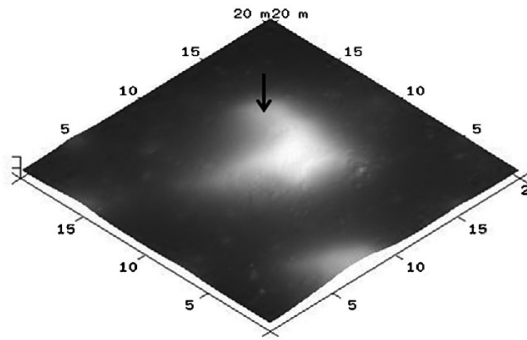

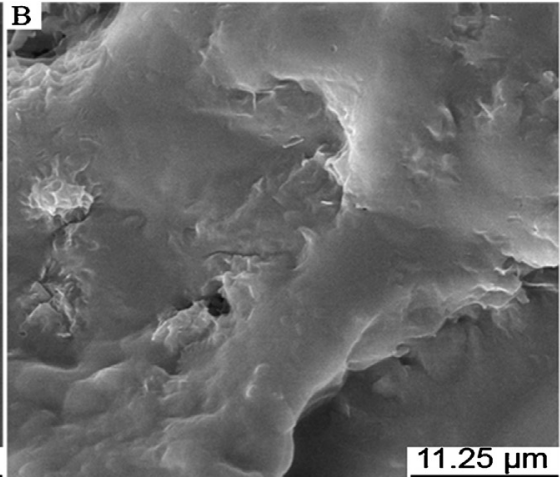

$\mathrm{E}$

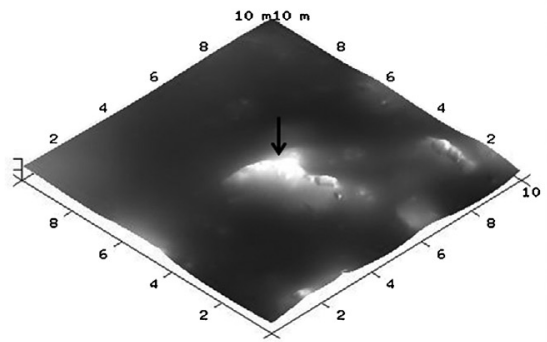

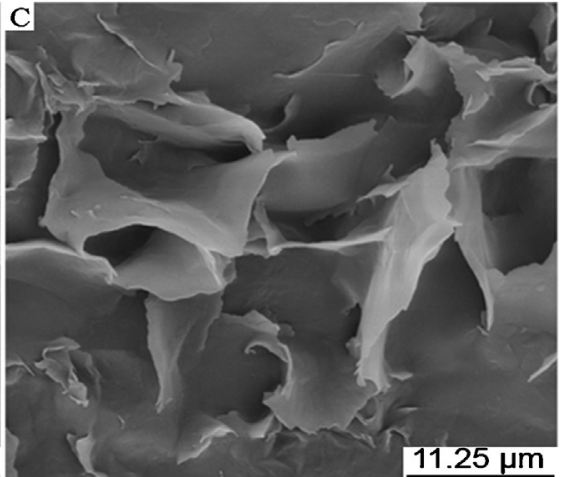

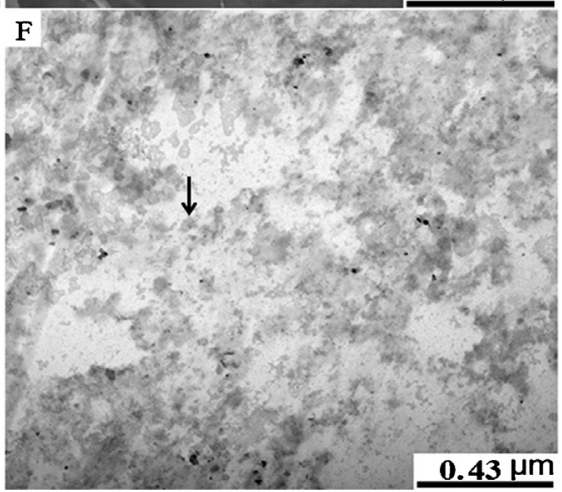

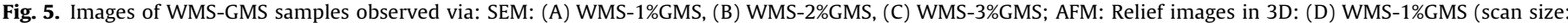
$20 \mu \mathrm{m} \times 20 \mu \mathrm{m}$ ), (E): WMS-3\%GMS (scan size: $20 \mu \mathrm{m} \times 20 \mu \mathrm{m}$ ); TEM: (F) WMS-1\%GMS.

Biliaderis, Page, Slade, \& Sirett (1985) reported that cooling curves are associated with helix organization of starch-lipid complexes in a semi-crystalline supramolecular structure. These molecular aggregates that form during the cooling period can exhibit perfection in crystalline domains, given the fact that it is a process that occurs at a much lower velocity. Eliasson (1994) confirmed that the crystals that form during the cooling period are thermally stronger than that of the first heating period and occur at a temperature that is lower than that of the first heating period.

The increased GMS concentration caused an $8.9 \%$ reduction in the $\Delta \mathrm{H}_{\mathrm{Cx}}$ of the NMS-GMS complexes (from $-1.92 \mathrm{~J} / \mathrm{g}$ to $-1.75 \mathrm{~J} / \mathrm{g}$ ) (Fig. 2-II, A), as well as a $10 \%$ reduction in the $\Delta \mathrm{H}_{\mathrm{Cx}}$ of the HAMS-
GMS complexes (from $-3.09 \mathrm{~J} / \mathrm{g}$ to $-2.79 \mathrm{~J} / \mathrm{g}$ ) (Fig. 2-II, C). However, complexing intensity was small in WMS-1\%GMS (Fig. 2-II, B1), and at higher emulsifier concentrations, no transition peak was observed in the thermograms (Fig. 2-II, B2 and B3) as also observed in X-ray difractograms (Fig. 1, B2 and B3).

During the reheating process, the dissociation of the recrystallized complexes occurred at temperatures lower than those of the first heating period. There was a slight reduction in $\Delta \mathrm{H}_{\mathrm{cx}}$ (from 3.66 to $3.10 \mathrm{~J} / \mathrm{g}$ ) and an increase in dissociation temperature (from 96.10 to $101.78^{\circ} \mathrm{C}$ ) of the NMS-GMS complex when the emulsifier concentration increased from $1 \%$ to $3 \%$ (Fig. 2-III, A1-A3). On the other hand, there was no change in $\Delta \mathrm{H}_{\mathrm{cx}}$ or in dissociation temperatures 
Table 1

Roughness, height, and diameter of the inclusion complexes prepared with different GMS concentrations and starches with different amylose contents.

\begin{tabular}{|c|c|c|c|c|c|}
\hline Sample & Roughness (nm) & Minimum height (nm) & Maximum height (nm) & Minimum diameter $(\mu \mathrm{m})$ & Maximum diameter $(\mu \mathrm{m})$ \\
\hline NMS-1\%GMS & $20.01 \pm 0.35^{\mathrm{b}}$ & $248.09 \pm 0.92^{b}$ & $440.04 \pm 0.7^{b}$ & $5.60 \pm 0.31^{b}$ & $15.30 \pm 0.46^{\mathrm{b}}$ \\
\hline NMS-3\%GMS & $81.03 \pm 0.78^{\mathrm{a}}$ & $477.41 \pm 0.86^{\mathrm{a}}$ & $766.51 \pm 0.83^{a}$ & $11.07 \pm 0.44^{\mathrm{a}}$ & $23.24 \pm 0.31^{\mathrm{a}}$ \\
\hline WMS-1\%GMS & $32.30 \pm 0.65^{\mathrm{a}}$ & - & - & - & - \\
\hline WMS-3\%GMS & $34.00 \pm 0.90^{\mathrm{a}}$ & - & - & - & - \\
\hline HAMS-1\%GMS & $51.05 \pm 0.90^{\mathrm{b}}$ & $772.40 \pm 0.75^{\mathrm{b}}$ & $1541.67 \pm 0.49^{b}$ & $5.11 \pm 0.19^{b}$ & $13.37 \pm 0.31^{\mathrm{b}}$ \\
\hline HAMS-3\%GMS & $120.33 \pm 0.83^{a}$ & $2059.26 \pm 0.40^{a}$ & $2603.67 \pm 0.94^{\mathrm{a}}$ & $7.21 \pm 0.47^{a}$ & $15.34 \pm 0.33^{a}$ \\
\hline
\end{tabular}

Values with different letters in the same column, for each starch, are significantly different $(\mathrm{p} \leq 0.05)$.

of HAMS-GMS complexes as GMS concentration increased (Fig. 2III, C1-C3). These results indicate that the NMS-GMS complex was more affected at higher GMS concentrations due to the fact that less amylose was available to form complexes. A peak between 50 and $60^{\circ} \mathrm{C}$ was also noted; it referred to the self-associated GMS dissociation. This peak was more clearly noted in the NMS-3\%GMS complex (Fig. 2-III, A3).

\subsection{Microscopic analysis}

Regardless of the emulsifier concentration, SEM images of the NMS-GMS complexes displayed faceted structures, which are characteristics of crystals that are embedded in the matrix (Fig. 3A-C).However, these faceted structures were found to be more strongly associated or stuck together, particularly in the NMS-1\%GMS and NMS-2\%GMS complexes (Fig. 3A and B). Similar structures were observed by Exarhopoulos \& Raphaelides (2012) in inclusion complexes prepared with normal maize and pea starches and different saturated fatty acids. Similar structures were also reported by Zabar et al. (2010) in a study on potato amylose complexed with stearic acid. As observed before, the thermal properties of starch-GMS complexes have shown the existence of polymorphic crystals, which have characteristic dissociation temperatures. These complexes are formed as insoluble precipitates in a neutral aqueous medium that exhibit crystalline faceted forms with a V-type pattern (Karkalas et al., 1995; Lesmes et al., 2009; Tang \& Copeland, 2007), as was also observed from the X-ray diffraction.

Atomic force microscopy was applied to help elucidate the starch-GMS inclusion complex structure on the micro and nano scales. Three-dimensional relief images of the NMS-GMS complexes (Fig. 3D and E) displayed a tendency on the part of the crystals to aggregate and form agglomerates of various sizes. When $1 \%$ GMS was used, these agglomerates had diameters from 5 to $15 \mu \mathrm{m}$, and heights from 248 to $440 \mathrm{~nm}$ (Fig. 3D $\downarrow$ ), besides a surface roughness of $20.0 \mathrm{~nm}$ (Table 1 ). As the GMS concentration increased (3\%), the diameter and height of the agglomerates also increased and measured from 11 to $23 \mu \mathrm{m}$ and from 477 to $766 \mathrm{~nm}$, respectively (Fig. 3E $\downarrow$ ). Meanwhile, surface roughness increased to $81.0 \mathrm{~nm}$ (Table 1). The increase in diameter, height, and roughness of the crystal agglomerates may be due to the self-association of GMS molecules that may have resulted in the over estimation of these parameters. In addition, the NMS-3\%GMS complex (Fig. 3E) also displayed a gelatinized mass surrounding the agglomerates suggesting the presence of gelatinized amylose chains that may be not complexed with GMS.

When the NMS-3\%GMS complex was observed at ahigher resolution (scan at $2.0 \mu \mathrm{m} \times 2.0 \mu \mathrm{m}$ ), the 3D relief images from AFM (Fig. 4A) revealed small particles that were few nanometers of diameter (from $80 \mathrm{~nm}$ to $150 \mathrm{~nm}$ ). These particles corresponded to crystals organized into packed lamellae in spheroid form that tended to self-aggregate in larger agglomerates. These results agree with previous reports by Lesmes et al. (2009) and Zabar et al. (2010). The phase image of the NMS-3\%GMS complex confirmed the presence of a gelatinized mass (Fig. 4B) that may consist of the lowest gelatinized amylose chains that could not host the GMS molecules. This result agrees with those obtained from DSC (Fig. 2-I, A3).

TEM images of the all starches complexed with $1 \%$ GMS displayed an aggregated strand structure interwoven with the emulsifier suggesting that the amylose chains randomly surrounded the GMS molecules (Figs. 3, 5, 6 F) Richardson et al. (2004) reported a similar behavior when investigating complexes prepared with maize, wheat, and potato starches and mixtures of polyglycerol ester and monoglyceride (PGE/MG) or GMS. These authors also reported that complex formation was more extensive depending on the emulsifier-to-starch ratio. The NMS-1\%GMS complex had short and closed strand structure and formed crystalline aggregates typical of the inclusion complex (Fig. 3F $\downarrow$ ).

SEM images showed that only the WMS-1\%GMS sample (Fig. 5A) displayed a faceted structure that seemed to be soaked in a shapeless gelatinized mass. Meanwhile, a bulk phase, and not the typically faceted structures, was observed in the WMS-2\%GMS and WMS-3\%GMS samples (Fig. 5B and C). Indeed, X-ray diffractograms and DSC curves of the WMS-GMS samples confirmed only inclusion complex formation between WMS and $1 \%$ emulsifier. These results indicate that the lower complexing ability of WMS was due to the limited amounts of amylose and long branch chains of amylopectin able to complex with GMS when compared to the amount of amylose available in NMS and HAMS. In a previous study, Garcia \& Franco (2015) reported the in vitro formation of inclusion complexes prepared with waxy maize starch and GMS. There was interaction between the few amylose molecules and/or longer branch chains of amylopectin of waxy maize starch and GMS. The AFM 3D relief images of WMS-GMS samples (Fig. 5D and E $\downarrow$ ) displayed a shapeless gelatinized mass. Neither the diameters nor the heights of the agglomerates could be calculated, regardless of the GMS concentration. The surface roughness of the WMS-GMS agglomerates was low and remained unchanged, even as the GMS concentration increased $(\sim 33 \mathrm{~nm}$; Table 1$)$. This result suggests that the surface roughness was the gelatinized shapeless mass that covered the WMS-GMS complexes.

Contrary to the NMS-1\%GMS complex, which exhibited short chains and a closed strand structure when observed in TEM, the WMS-1\%GMS complex displayed more open chains and various uncomplexed GMS molecules (Fig. 5F $\downarrow$ ). During complexation, the uncomplexed molecules of the WMS were also deformed, and the amylopectin that had leaked out formed a gelatinized shapeless mass. These findings were also revealed from SEM and AFM and indicate that less complexing occurred between few amylose and/or long branch chains of amylopectin with GMS.

Finally, the HAMS-GMS complexes observed in SEM (Fig. 6A-C) displayed faceted structures, which became better defined as the GMS concentration increased. This change was likely due to the larger amount of amylose available for complex formation.This result suggests that, in this case, concentrations less than $3 \%$ GMS were not high enough to interact with all of the amylose available for complex formation, as was already observed in the results obtained from DSC and X-ray diffraction.The HAMSGMS complexes did not change the $\mathrm{t}_{\mathrm{cx}}$ or $\Delta \mathrm{H}_{\mathrm{cx}}$ parameters, and they exhibited diffraction peaks with increasingly clear resolutions 

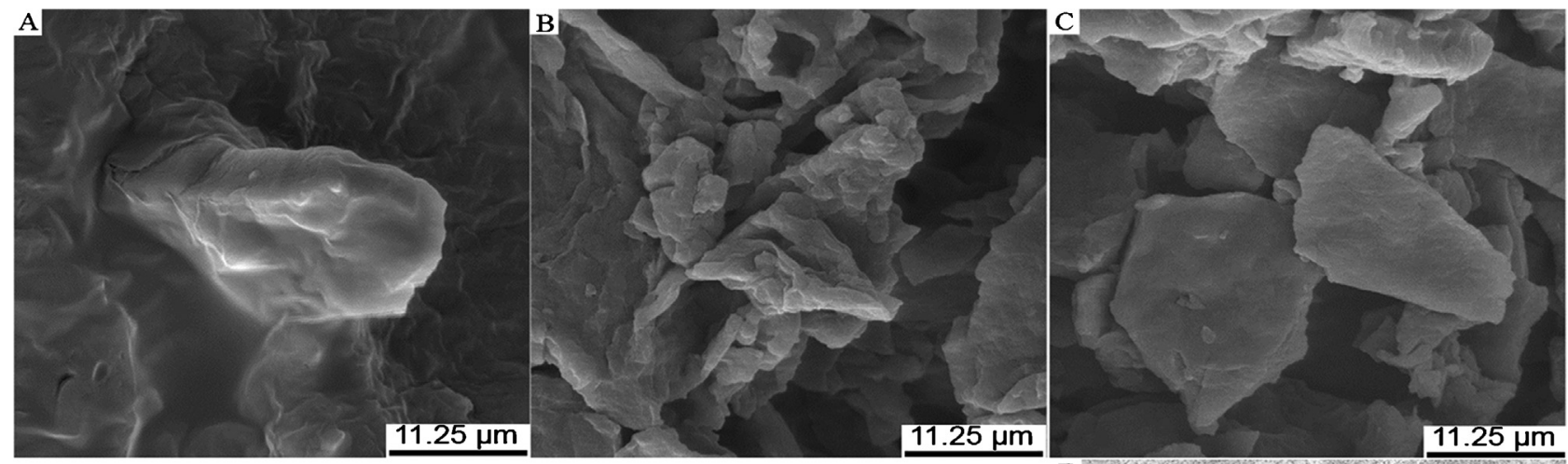

D

$\mathrm{E}$

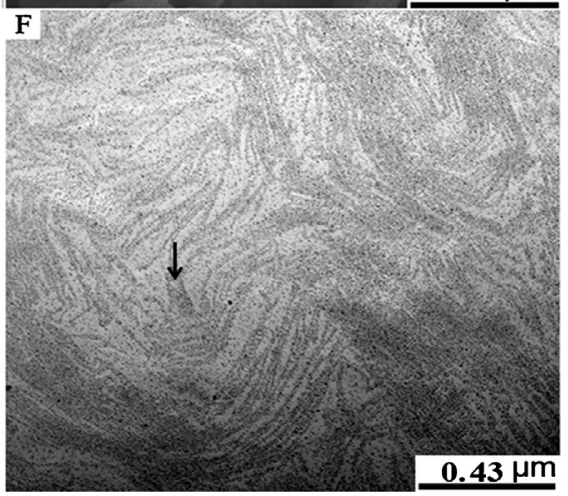

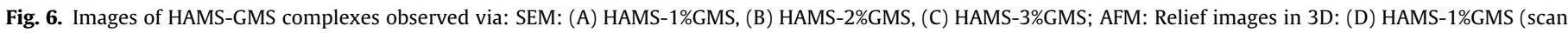
size: $40 \mu \mathrm{m} \times 40 \mu \mathrm{m}$ ); (E): HAMS-3\%GMS (scan size: $40 \mu \mathrm{m} \times 40 \mu \mathrm{m}$ ); TEM: (F) HAMS-1\%GMS.

as the GMS concentration increased. In the HAMS-1\%GMS complex, protrusions appeared to exit from the partially gelatinized granule mass. Richardson et al. (2004) also observed these protrusions when analyzing amylomaize starch-fatty acid systems. These authors determined these protrusions to be amylose molecules which had tried to exit from the granules and which had exit only partially. The molecules immediately interacted with available stearate or myristate ions present in the medium to form an inclusion complex.

The AFM 3D images of the HAMS-GMS complexes also revealed crystal aggregates. However, no excess of emulsifier close to the crystal agglomerates was observed, even in the HAMS-3\%GMS complex. The HAMS-1\%GMS crystal agglomerates had diameters of 5 to $13 \mu \mathrm{m}$, heights of 772 to $1541 \mathrm{~nm}$, and a surface roughness of $51 \mathrm{~nm}$ (Fig. 6D $\downarrow$; Table 1). As the GMS concentration increased, these parameters increased to $7-15 \mu \mathrm{m}$, to $2059-2603 \mathrm{~nm}$, and to $120 \mathrm{~nm}$, respectively (Fig. $6 \mathrm{E}_{\downarrow}$; Table 1 ). The HAMS-GMS complexes were found to have greater heights and more roughness than the NMS-GMS complexes. On the other hand, the diameters of the HAMS-GMS complexes were slightly lower than those of the NMS-GMS complexes due to the lower amount of GMS molecules available for self-association.

The HAMS-1\%GMS complex observed under TEM displayed thicker and longer strand structures than those observed in the NMS-1\%GMS complex due to the high concentration of amylose chains that aggregated to form inclusion complex with GMS (Fig. $6 \mathrm{~F} \downarrow$ ). These results reflect the higher complexing capacity of this starch and GMS, results which are consistent with those obtained from SEM and AFM. Complexation of amylose and GMS was more intense in HAMS, followed by the NMS and WMS. These results suggest that both the amount and the length of the amylose chains directly influence the ability of amylose to form complexes with lipids. Lee, Han, \& Lim (2009) and Takeda, Takeda, \& Hizukuri (1989) reported that the amylose chains in high amylose maize starch were smaller and less branched than those in normal maize starch. The smaller sized amylose chains might have greater mobility and the less branched structure might provide more chance for the chains to associate (Lee et al., 2009).

\section{Conclusions}

Inclusion complexes formed between GMS and maize starches with different amylose contents display V-type crystalline patterns with IIb-type polymorphism, including those formed with few amylose molecules and/or longer branch chains of amylopectin of WMS and 1\%GMS. The SEM images of the NMS-GMS, WMS-GMS and HAMS-GMS complexes displayed faceted structures, which are characteristic of crystals. AFM images revealed the starch-GMS inclusion complex structure on micro and nano scales, as well as the tendency of the crystals to aggregate and form agglomerates of various sizes. TEM images showed an aggregated strand structure interwoven with the GMS. Emulsifier and amylose quantities directly influence the formation of these complexes. Above critical micellar concentrations, GMS is more likely to self-associate than to form complexes with amylose.

\section{Acknowledgments}

The authors would like to thank the São Paulo State Research Foundation - FAPESP -(Grants No. 2011/20387-1 and No. 2010/18557-3) and National Council for Scientific and Technological Development - CNPQ - (Grants No. 306320/2012-6) in Brazil for financial support.

\section{References}

Biliaderis, C. G., Page, C. M., Slade, L., \& Sirett, R. R. (1985). Thermal behavior of amylose-complexes. Carbohydrate Polymers, 5, 365-389.

Carrillo-Navas, H., Pérez-Alonso, C., Fouconnier, B., Vernon-Carter, E. J., \& Alvarez-Ramírez, J. (2014). Inertial effects of adsorbed glycerol monostearate 
crystals on the shear rheology of water/canola oil interfaces. Journal of Food Engineering, 125, 112-118.

Chang, F., He, X., \& Huang, Q. (2013a). The physicochemical properties of sewelled maize starch granules complexed with lauric acid. Food Hydrocolloids, 32, 365-372.

Chang, F., He, X., \& Huang, Q. (2013b). Effect of lauric acid on the V-amylose complex distribution and properties of swelled normal corn starch granules. Journal of Cereal Science, 58, 89-95.

Cheetam, N. W. H., \& Tao, L. (1998). Variation in crystalline type with amylose content in maize starch granules: an X-ray powder diffraction study. Carbohydrate Polymers, 36, 277-284.

Conde-Petit, B., Escher, F., \& Nuessli, J. (2006). Structural features of starch-flavor complexation in food mode systems. Trends in Food Science and Technology, 17, 227-235.

Eliasson, A. C. (1994). Interactions between starch and lipids studied by DSC. Thermochimica Acta, 246, 343-356.

Exarhopoulos, S., \& Raphaelides, S. N. (2012). Morphological and structural studies of thermally treated starch-fatty acid systems. Journal of Cereal Science, 55 139-152.

Fanta, G. F., Felker, F. C., \& Shogren, R. L. (2002). Formation of crystalline aggregates in slowly-cooled starch solutions prepared by steam jet cooking. Carbohydrate Polymers, 48, 161-170.

Fanta, G. F., Felker, F. C., Shogren, R. L., \& Salch, J. H. (2006). Effect of fatty acid structure on the morphology of spherulites formed from jet cooked mixtures of fatty acids and defatted cornstarch. Carbohydrate Polymers, 66, 60-70.

Galloway, G. I., Biliaderis, C. G., \& Stanley, D. W. (1989). Properties and structure of amylose-glycerol monostearate complexes formed in solution or on extrusion of wheat-flour. Journal of Food Science, 54, 950-957.

Garcia, M. C., \& Franco, C. M. L. (2015). Effect of glycerol monostearate on the gelatinization behavior of maize starches with different amylose contents. Starch/Starke, 66, 1-10.

Gelders, G. G., Vanderstukken, T. C., Goesaert, H., \& Delcour, J. A. (2004). Amylose-lipid complexation: a new fractionation method. Carbohydrate Polymers, 56, 447-458.

Ghosh, S., \& Rousseau, D. (2009). Freeze-thaw stability of water-in-oil emulsion. Journal of Colloid and Interface Science, 339, 91-102.

Godet, M. C., Bizot, H., \& Buléon, A. (1995). Crystallization of amylose-fatty acid complexes prepared with different amylose chain lengths. Carbohydrate Polymers, 27, 47-52.

Godet, M. C., Bouchet, B., Colonna, P., Gallant, D. J., \& Buléon, A. (1996). Crystalline amylose-fatty acid complexes: morphology and crystal thickness. Journal of Food Science, 61, 1196-1201.

Karkalas, J., Ma, S., Morrison, W. R., \& Pethrick, R. A. (1995). Some factors determining thermal properties of amylose inclusion complexes with fatty acids. Carbohydrate Research, 268, 233-247.

Kawai, K., Takato, S., Sasaki, T., \& Kajiwara, K. (2012). Complex formation, thermal properties: and in-vitro digestibility of gelatinized potato starch-fatty acid mixtures. Food Hydrocolloids, 27, 228-234.

Lalush, I., Bar, H., Zakaria, I., Eichler, S., \& Shimoni, E. (2005). Utilization of amylose-lipid complexes as molecular nanocapsules for conjugated linoleic acid. Biomacromolecules, 6, 121-130.
Lee, J. H., Han, J.-A., \& Lim, S.-T. (2009). Effect of pH on aqueos structure of maize starch analyzed by HPSEC-MALLS-RI system. Food Hydrocolloids, 23, 1935-1939.

Lesmes, U., Chen, S. H., Shener, Y., \& Shimoni, E. (2009). Effects of long chain fatty acid unsaturation on the structure and controlled release properties of amylose complexes. Food Hydrocolloids, 23, 667-675.

Mira, I., Persson, K., \& Villwock, V. K. (2007). On the effect of surface active agents and their structure on the temperature-induced changes of normal and waxy wheat starch in aqueous suspension. Part 1. Pasting and calorimetric studies. Carbohydrate Polymers, 68, 665-678.

Morrison, W. R., Law, R. V., \& Sanape, C. E. (1993). Evidence for inclusion complexes of lipids with $\mathrm{V}$-amylose in maize: rice and oat starches. Journal of Cereal Science, 18, 107-109.

Nara, S., \& Komiaya, T. (1983). Studies on the relationship between water-satured state and crystallinity by the diffraction method for moistened potato stach. Starch/Starcke, 35, 407-410.

Putseys, J. A., Lamberts, L., \& Delcour, J. A. (2010). Amylose-inclusion complexes: formation, identity and physico-chemical properties. Journal of Cereal Science. $51,238-247$.

Putseys, J. A., Liesbeth, J. D., Lamberts, L., Goesaert, H., \& Delcour, J. A. (2009). Production of tailor made short chain amylose-lipid complexes using varying reaction conditions. Carbohydrate Polymers, 78, 854-861.

Richardson, G., Kidman, S., Langton, M., \& Hermansson, A. M. (2004). Differences in amylose aggregation and starch gel formation with emulsifiers. Carbohydrate Polymers, 58, 7-13.

Seo, T.-R., Kim, J.-Y., \& Lim, S.-T. (2015). Preparation and characterization of crystalline complexes between amylose and C18 fatty acids. Food Science and Technology, 64, 889-897.

Takeda, C., Takeda, Y., \& Hizukuri, S. (1989). Structure of amylomaize amylose. Cereal Chemistry, 66, 22-25.

Tang, M. C., \& Copeland, L. (2007). Analysis of complexes between lipids and wheat starch. Carbohydrate Polymers, 67, 80-85.

Vasiliadou, E., Rapphaelides, S. N., \& Papastergiadis, E. (2015). Effect of heating time and temperature on partially gelatinized starch-fatty acid interactions. Food Science and Technology, 60, 698-707.

Villwock, V. K., Eliasson, A. C., Silverio, J., \& Bemiller, J. N. (1999). Starch-lipid interactions in common, waxy, ae du: and ae su2 maize starches examined by Differential Scanning Calorimetry. Cereal Chemistry, 76, 292-298.

Zabar, S., Lesmes, U., Katz, I., Shimoni, E., \& Bianco-Peled, H. (2009). Studying different dimensions of amylose-long chain fatty acid complexes: molecular: nano and micro level characteristcs. Food Hydrocolloids, 23, 1918-1925.

Zabar, S., Lesmes, U., Katz, I., Shimoni, E., \& Bianco-Peled, H. (2010). Structural characteriztion of amylose-long chain fatty acid complexes produced via the acidification. Food Hydrocolloids, 24, 347-357.

Zhang, B., Huang, Q., Luo, F. X., \& Fu, X. (2012). Structural characterizations and digestibility of debranched high-amylose maize starch complexed with lauric acid. Food Hydrocolloids, 28, 174-181.

Zhou, X., Ren, W., Zhang, Y., Yoo, S.-H., \& Lim, S.-T. (2013). Effects of amylose chain length and heat treatment on amylose-glycerol monocaprate complex formation. Carbohydrate Polymers, 95, 227-232. 